\title{
Le statut juridique de l'animal : perspectives comparatives
}

Muriel Falaise

Maître de conférences de droit privé

Université Lyon 3 France

S'il est une époque qui semble en passe d'être révolue, c'est bien celle au cours de laquelle on pensait que l'animal ne pouvait être appréhendé qu'en sa qualité de bien au service de l'homme. Un nombre croissant de découvertes scientifiques notamment en biologie, éthologie, neuroscience, éthique animale ont permis d'établir sans conteste la propension de l'animal à ressentir douleurs et émotions (empathie, peur, stress, joie,...). Dés lors, comment s'affranchir de la problématique concernant la place qui leur est accordée au sein de nos sociétés ? L'évolution du regard porté sur la condition animale, la multiplication des actions militantes en faveur des animaux ont conduit certains législateurs à s'emparer de la question du positionnement juridique de l'animal. C'est dans ce contexte que le 4 décembre 2015, l'Assemblée nationale du Québec a adopté à l'unanimité la Loi visant l'amélioration de la situation juridique de ''animal $^{1}$ constituée d'une première partie consacrant la modification générale du statut des animaux et d'une seconde partie renforçant les règles applicables en matière de bien-être et de sécurité de l'animal. Cette nouvelle législation permet-elle pour autant au Québec de s'inscrire dans un mouvement mondial favorisant l'émergence d'un nouveau paradigme juridique ?

\section{I - L'adoption de la loi du 4 décembre 2015 dans un contexte favorable à la protection animale}

La détermination du statut juridique de l'animal et l'élaboration du régime juridique qui lui est applicable émanent de sources juridiques diverses selon les pays.

\footnotetext{
${ }^{1}$ RLRQ, chapitre 35.
} 
Dans plusieurs Etats, des principes de respect et de protection de l'animal ont été intégrés à la Constitution. Ce positionnement au sein de la norme juridique suprême de l'Etat assure à l'animal une place spécifique dans la société. Sept pays dans le monde ont fait ce choix : l'Allemagne, l'Argentine, l'Autriche, le Brésil, l'Egypte, l'Inde et la Suisse. La portée de ces dispositions constitutionnelles varie d'un Etat à l'autre dans la mesure où elles ne s'appliquent pas toutes aux mêmes catégories d'animaux et ne fixent pas les mêmes principes. A ce jour, seules les constitutions égyptienne et suisse font explicitement référence à l'animal en sa qualité d'être vivant et les constitutions autrichienne et égyptienne assurent un traitement respectueux de son bien-être. Mais quelque soit le principe énoncé, inscrire des valeurs propres à l'animal au sein d'une Constitution présente un avantage majeur : celui de prendre en considération ses intérêts $a$ priori dés lors qu'un projet de loi le concernant est examiné.

A défaut de modifications constitutionnelles, l'évolution se traduit par l'adoption de dispositions législatives donc certaines font l'objet d'une codification. On les retrouve parfois disséminées dans de nombreux codes à l'instar de la France où les règles applicables aux animaux figurent dans neuf codes différents ${ }^{2}$. Il n'en demeure pas moins que l'insertion de dispositions au sein du code civil doit être considérée comme un signal fort dans la mesure où il constitue le droit commun et a donc vocation à fixer le cadre général du statut des animaux et à s'appliquer à l'ensemble des animaux.

Au Canada, les trois paliers gouvernementaux (municipal, provincial et fédéral) se répartissent les compétences en matière de protection et de bien-être animal. Il en résulte un patchwork de dispositions nationales et locales d'où une importante disparité entre les provinces. Le rapport annuel publié depuis 2008 par Animal Legal Defense Fund (ALDF)

\footnotetext{
${ }^{2}$ Code civil, code rural et de la pêche maritime, code de l'environnement, code pénal, code de procédure pénale, code général des collectivités territoriales, code de la santé publique, code de l'action sociale et des familles, code de la sécurité intérieure.
} 
donne un classement des provinces à partir d'une analyse comparative détaillée des lois de protection animale en vigueur dans les 13 provinces et territoires canadiens. En juillet 2017, l'Île-du-Prince-Édouard (1), le Manitoba (2), la Nouvelle-Écosse (3) et le NouveauBrunswick (4) sont les provinces les plus protectrices. A l'inverse, le Nunavut (13), les Territoires du Nord-Ouest (12), la Saskatchewan (11) et l'Alberta (10), se révèlent être les provinces où la maltraitance animale est la moins sévèrement sanctionnée. Quant aux autres provinces, elles se classent respectivement $5^{\text {ème }}$ (Colombie-Britannique), $6^{\text {ème }}$ (Ontario), $7^{\text {ème }}$ (Terre-Neuve et Labrador), $8^{\text {ème }}$ (Québec) et $9^{\text {ème }}$ (Yukon) ${ }^{3}$. Ce classement évolue au gré des avancées législatives et c'est ainsi que le Québec est passé de la peu enviable $12^{\text {ème }}$ position en 2015 à la $6^{\text {ème }}$ position en 2016 suite à l'adoption de la Loi visant l'amélioration de la situation de l'animal ${ }^{4}$. Jusqu'à cette loi, la protection animale était encadrée par la Loi sur la protection sanitaire des animaux (section IV.1.1)et par le Code criminel. L'adoption de ce texte a provoqué deux changements majeurs dans la province québécoise : la modification du code civil et la création de la Loi sur le bien-être et la sécurité de l'animal.

\section{II - Les dispositions du code civil}

Depuis, le 4 décembre 2015, date d'entrée en vigueur de la $1 \mathrm{oi}^{5}$, un nouvel article 898.1 a été inséré au code civil québécois :

«Les animaux ne sont pas des biens. Ils sont des êtres doués de sensibilité et ils ont des impératifs biologiques.

Outre les dispositions des lois particulières qui les protègent, les dispositions du présent code et de toute autre loi relative aux biens leur sont néanmoins applicables. »

Ces deux alinéas qui modifient la situation juridique de l'animal ont largement été inspirés par la tendance réformatrice qui conduit à distinguer l'animal des choses et

\footnotetext{
${ }^{3}$ https://aldf.org/wp-content/uploads/2018/06/2017-Canadian-Rankings-Report-1.pdf

${ }^{4} \mathrm{http}: / /$ acracq.com/Documents/2016-Canadian-Rankings-Report.pdf

${ }^{5} \mathrm{La}$ date d'entrée en vigueur est fixée par l'article 10, à l'exception des articles 16 à 20 relatifs à la possession d'un permis pour la détention de certaines catégories ou nombres d'animaux.
} 
parfois des biens. C'est en 1988 que ce mouvement a débuté en Europe avec l'adoption par le législateur autrichien de l'article §285a du code civil disposant que «Les animaux ne sont pas des choses; ils sont protégés par les lois spéciales » ${ }^{6}$. Plusieurs législateurs ont par la suite adopté des dispositions similaires. C'est le cas en Allemagne ${ }^{7}$, Azerbadjan $^{8}$, Catalogne $^{9}$, Colombie $^{10}$, France $^{11}$, Moldavie $^{12}$, Pays-Bas ${ }^{13}$, Portugal ${ }^{14}$, République Tchèque ${ }^{15}$ et Suisse ${ }^{16}$. La Belgique et l'Espagne ont également récemment initié un projet de réforme de leur code civil. Une analyse comparative des dispositions contenues dans les différents codes civils fait apparaitre deux tendances. Un premier groupe est constitué par les codes qui se limitent à opérer une distinction entre l'animal et les biens. C'est notamment le cas du code civil suisse, qui comme les codes autrichien et allemand, a été modifié afin faire sortir l'animal de la catégorie des choses inanimées et de le protéger par des dispositions spécifiques. Un second groupe est composé de codes civil qui ont connu une évolution plus récente et procédé à une réforme plus profonde en consacrant la qualité d'être vivant sensible de l'animal. Dans cette seconde catégorie figure la Colombie, la France, le Québec, le Portugal et la République Tchèque.

Malgré l'exclusion de l'animal de la catégorie des biens, tous les législateurs ont tour à tour manqué de clairvoyance car comment peut-on à la fois être et ne pas être ? Ne pas être un bien et être toutefois soumis au régime de la propriété ! Tous les codes civils consacrant la sortie de l'animal de la catégorie des biens maintiennent l'application des règles relatives aux choses sauf dispositions contraires. Cette dichotomie est une

\footnotetext{
${ }^{6}$ Loi fédérale du 10 mars 1988.

${ }^{7}$ Article $\$ 90$ a du code civil allemand (BGB) issu de la loi du 20 août 1990.

${ }^{8}$ Art 135-3 du code civil d'Azerbaïdjan.

${ }^{9}$ Article 511-1 du code civil de Catalogne issu de la loi 5/2006 du 10 mai 2006.

${ }^{10}$ Article 655 du code civil issu de la loi no 1774 du 6 janvier 2016.

${ }^{11}$ Article 515-14 du code civil issu de la loi n²015-177 du 16 février 2015.

${ }^{12}$ Art. 287 du code civil moldave.

${ }^{13}$ Article $3: 2 \mathrm{a}: 1$ issu de la loi du 19 mai 2011.

${ }^{14}$ La loi $\mathrm{n}^{\circ} 8 / 2017$ du 3 mars 2017 instaure un statut propre aux animaux, reconnaissant leur nature en tant qu'êtres sensibles.

${ }_{15}^{15}$ Article 494 du code civil issu de la loi du 3 janvier 2012.

${ }^{16}$ La Suisse a modifié le statut juridique de l'animal le 4 octobre 2002 en insérant un nouvel article 641a au code civil.
} 
conséquence de la structure même du code civil qui n'opère de classifications qu'entre deux catégories : les personnes et les biens. Faute d'une autre catégorie juridique, les animaux demeurent donc dans la partie relative aux biens. Il en résulte une fiction juridique qui conduit le législateur à affirmer dans un même article que l'animal n'est pas un bien mais qu'il reste soumis au régime des biens corporels. Le régime juridique ne suit donc pas la qualification d'être doué de sensibilité. L'organisation des articles au sein des différents livres du code civil maintien une incohérence. Le nouvel article 898.1 du code civil québécois a ainsi été inséré dans le livre quatrième consacré aux biens, à l'instar des modifications effectuées au sein des autres codes civils. Seul le code civil portugais, modifié suite à l'adoption de la loi du 3 mars 2017, fait figure d'exception. Les trois articles relatifs aux animaux êtres vivants sensibles ${ }^{17}$ ont été intégrés dans le titre traitant «Des relations juridiques » sous le Sous-titre I-A «Des animaux » qui figure lui-même dans le Sous-titre I «Des personnes» et non pas dans le Sous-titre II « Des choses». Cette structure distingue toujours deux catégories, les personnes d'une part et les choses d'autre part, mais la grande nouveauté réside dans l'insertion de l'animal dans la partie relative aux personnes.

La mise en place du mécanisme d'exclusion par principe des animaux de la catégorie des biens tout en maintenant leur régime à l'identique de celui des autres biens ne peut cependant pas être assimilé à un simple toilettage. Il constitue une étape fondamentale car désormais, les lois et les règlements sont interprétés par les tribunaux en fonction de ces dispositions. La reconnaissance dans le droit commun de la qualité d'être vivant sensible de l'animal ouvre la voie à des évolutions jurisprudentielles. Deux jurisprudences récentes rendues de part et d'autre de l'Atlantique confirment cette tendance. Si les juges français

\footnotetext{
${ }^{17}$ Article 201. ${ }^{\circ}-\mathrm{B}:$ «Les animaux sont des êtres vivants dotés d'une sensibilité et font l'objet d'une protection juridique selon leur nature. »

Article $201 .^{\circ}-\mathrm{C}:$ "La protection légale des animaux est assurée par les dispositions du présent code et de la législation spéciale. »

Article 201. ${ }^{\circ}-\mathrm{D}:$ : En l'absence d'une loi spéciale, les dispositions relatives aux choses s'appliquent de manière subsidiaire aux animaux, pour autant qu'elles ne soient pas incompatibles avec leur nature. »
} 
indemnisaient déjà depuis fort longtemps le préjudice affectif résultant de la perte d'un animal $^{18}$, la reconnaissance de la sensibilité animale au sein du code civil a ouvert d'autres voies comme en témoigne l'arrêt rendu par la $1^{\text {ère }}$ chambre civile de la Cour de Cassation le 9 décembre $2015^{19}$. Dans cette affaire, qui portait sur un classique défaut de conformité d'un chiot vendu par une éleveuse, la Cour de Cassation a confirmé la position des juges du fond qui avaient retenu que l'attachement de la demanderesse pour son chien ne permettait pas le remplacement du celui-ci à titre de réparation comme le proposait la défenderesse. Les juges ont retenu que «le chien en cause était un être vivant, unique et irremplaçable, et un animal de compagnie destiné à recevoir l'affection de son maître, sans aucune vocation économique ». Les juges québécois se sont à leur tour saisis de la qualité d'être vivant sensible de l'animal dans une décision rendue le 16 janvier 2017 par la chambre civile de la Cour du Québec ${ }^{20}$. En l'espèce la propriétaire d'un chien avait confié son animal à un chenil duquel il s'était échappé avant d'être heurté mortellement par une voiture. La tribunal a retenu la responsabilité de la défenderesse, propriétaire du chenil, et l'a condamnée d'une part à rembourser la valeur d'acquisition du chien au moment du décès et d'autre part à verser des dommages et intérêts au titre du préjudice moral en rappelant que depuis l'entrée en vigueur de l'article 898.1 du code civil l'animal n'est plus considéré comme un simple bien mais comme un être vivant doué de sensibilité.

En intégrant ensuite au sein de l'article 898.1 la notion d'impératifs biologiques, le législateur québécois a franchi un pas supplémentaire vers la reconnaissance d'une personnalité juridique spécifique au profit de l'animal. C'est en effet le premier code à faire explicitement référence aux besoins de l'animal. Il ouvert une nouvelle voie qui a été

\footnotetext{
${ }^{18}$ Dans ce sens, voir l'arrêt rendu par la $1^{\text {ère }}$ chambre civile de la cour de cassation le 16 janvier 1962.

${ }^{19}$ Cassation civile 1ère, 9 décembre 2015, 14-25910.

${ }^{20}$ Lavigne c. Brousseau-Masse (Chenil Moya), 2017 QCCQ 503 (CanLII).
} 
suivie par le législateur portugais qui, a l'occasion de la récente réforme du code civil issue de la loi du 3 mars 2017, a adopté deux articles novateurs :

- l'article 1305A qui fixe l'obligation pour tout propriétaire d'assurer le bien-être de son animal et précise les moyens pour y parvenir ${ }^{21}$;

- l'article 1793A qui organise les modalités de garde de l'animal en cas de divorce ${ }^{22}$.

\section{III -Les dispositions relatives au bien-être et à la sécurité de l'animal}

La Loi sur le bien-être et la sécurité de l'animal ${ }^{23}$ modifie la section IV.1.1 de la Loi sur la protection sanitaire des animaux ${ }^{24}$, le Règlement sur les animaux en captivitée ${ }^{25}$ et abroge le Règlement sur les espèces ou catégories d'animaux ${ }^{26}$. Elle établit ainsi dans un seul texte les exigences minimales relatives au bien-être et à la sécurité des animaux. La finalité est d'assurer un meilleur traitement des animaux tout au long de leur vie en mettant à la charge des particuliers et des professionnels de nouvelles obligations. En comparaison des différentes législations nationales applicables en matière de bien-être et de protection animale, la loi québécoise interpelle par son contenu qui reflète des positions discordantes. En effet, si certaines mesures semblent peu significatives, d'autres à l'inverse peuvent être considérées comme avant-gardistes. Plusieurs éléments illustrent

\footnotetext{
${ }^{21}$ Article $1305 \mathrm{~A}$ :

"1. Le propriétaire d'un animal veille au bien-être de l'animal, respecte les caractéristiques de chaque espèce et observe, dans l'exercice de ses droits, les dispositions particulières relatives à l'élevage, la reproduction, la garde et la protection des animaux et à la sauvegarde des espèces menacées le cas échéant.

2. Aux fins du paragraphe précédent, l'obligation de garantir le bien-être comprend notamment: a) La garantie d'accès à l'eau et à la nourriture en fonction des besoins de l'espèce en question; b) la garantie d'accès aux soins médicaux et vétérinaires, le cas échéant, y compris les mesures prophylactiques, d'identification et de vaccination prévues par la loi.

3 - Le droit de propriété à l'égard d'un animal ne s'étend pas à la possibilité d'infliger des souffrances ou tout autre mauvais traitement sans raison valable, entraînant des souffrances injustifiées, l'abandon ou la mort. »

${ }^{22}$ Article 1793A : "Les animaux de compagnie sont confiés à l'un ou aux deux conjoints, compte tenu, entre autres, des intérêts de chacun des époux et des enfants du couple ainsi que du bien-être de l'animal ».

${ }^{23}$ RLRQ, chapitre B-3.1.

${ }^{24}$ RLRQ, chapitre P- 42.

${ }^{25}$ RLRQ, chapitre C-61.1, r. 5.

${ }^{26}$ RLRQ, chapitre P- 42, r. 6.
} 
ces atermoiements.

En premier lieu, les considérants sont fondamentaux car ils témoignent de la nouvelle place que le législateur entend accorder aux animaux au sein de la société québécoise et de la ligne de conduite qu'il se fixe :

«CONSIDÉRANT que la condition animale est devenue une préoccupation sociétale;

CONSIDÉRANT que les animaux contribuent à la qualité de vie de la société québécoise; CONSIDÉRANT que l'espèce humaine a une responsabilité individuelle et collective de veiller au bien-être et à la sécurité des animaux;

CONSIDÉRANT que l'animal est un être doué de sensibilité ayant des impératifs biologiques;

CONSIDÉRANT que l'État estime essentiel d'intervenir afin de mettre en place un régime juridique et administratif efficace afin de s'assurer du bien-être et de la sécurité de l'animal. »

En second lieu, l'article premier limite le champ d'application de ce texte à certaines catégories d'animaux contrairement à l'article 898-1 nouveau du code civil qui est d'application générale. La loi vise spécifiquement les animaux domestiques ${ }^{27}$, les animaux de compagnie ${ }^{28}$ et les équidés tout en n'excluant pas que d'autres animaux puissent y relever par voie réglementaire. Les animaux sauvages demeurent régis par la Loi sur la conservation et la mise en valeur de la faune ${ }^{29}$.

En troisième lieu, certaines dispositions du chapitre II relatif à l'obligation de soins et aux actes interdits appellent des commentaires, notamment les articles 5 et 6 qui sont

\footnotetext{
${ }^{27}$ Au titre des animaux domestiques relèvent du champ d'application de cette loi tout animal domestique ou sauvage ou poisson captifs élevés pour la production de produits d'origine animale (viande, fourrure, laine...).

${ }^{28}$ Il s'agit de tout animal domestique ou sauvage vivant au foyer de l'homme en tant que compagnon et à des fins d'agrément.

${ }^{29}$ RLRQ, c. C-61.1.
} 
fondamentaux dans l'organisation de la protection juridique de l'animal et qui font l'objet de vives critiques car de nombreuses activités professionnelles se trouvent exclues de leur champ d'application sans aucune justification (agriculture, médecine vétérinaire, enseignement et recherches scientifiques ${ }^{30}$ ). L'article 5 impose notamment au propriétaire ou gardien de l'animal une obligation de soins propres à ses impératifs biologiques afin de ne pas compromettre son bien-être et sa sécurité. Le législateur y définit la notion d'impératifs biologiques ${ }^{31}$ en faisant référence aux cinq libertés destinées à protéger l'intégrité physique et mentale de l'animal telles que retenues par l'OIE (organisation mondiale de la santé animale $)^{32}$. Des dispositions similaires sont d'ailleurs présentes dans plusieurs législations nationales notamment au Royaume-Uni ${ }^{33}$, Pays-Bas ${ }^{34}$, Grèce ${ }^{35}$ et Colombie $^{36}$. Quant à l'article 6, il définit et interdit toute situation de détresse causée à un animal par l'effet d'un acte ou d'une omission.

Avec l'article 8 relatif à la stimulation, la socialisation et l'enrichissement du milieu le

\footnotetext{
${ }^{30}$ Ces exclusions sont prévues par l'article 7 de la loi.

${ }^{31}$ Article 1, 5०: «Les besoins essentiels d'ordre physique, physiologique et comportemental liés, notamment, à l'espèce ou la race de l'animal, à son âge, à son stade de croissance, à sa taille, à son niveau d'activité physique ou physiologique, à sa sociabilité avec les humains et autres animaux, à ses capacités cognitives, à son état de santé, ainsi que ceux liés à son degré d'adaptation au froid, à la chaleur ou aux intempéries. »

${ }^{32}$ Les 5 libertés définies par l'OIE consistent à :

1- Ne pas souffrir de faim ou de soif

L'animal doit avoir accès à de l'eau fraîche et à une nourriture adaptée en quantité suffisante qui lui assure une bonne santé et de la vigueur.

2 - Ne pas souffrir d'inconfort

L'animal doit avoir un accès libre à un environnement approprié comportant au minimum un abri et une aire de couchage.

3- Ne pas souffrir de douleurs, de blessures ou de maladies

L'animal doit bénéficier d'une prévention ou d'un diagnostic vétérinaire rapide suivi d'un traitement adapté.

4 - Pouvoir exprimer les comportements naturels propres à l'espèce

L'animal doit disposer d'un espace suffisant, d'un environnement approprié et de la possibilité de contacts et d'interaction avec d'autres membres de son espèce.

5 - Ne pas éprouver de peur ou de détresse

Les conditions d'élevage et les pratiques ne doivent pas induire de souffrances psychologiques.

${ }^{33}$ Article de la loi du 8 novembre 2006.

${ }^{34}$ Article 1.3.3 de la loi du 19 mai 2011.

${ }^{35}$ Article 1 de la loi du 2 février 2012.

${ }^{36}$ Article 3 de la loi du 6 janvier 2016.
} 
législateur québécois a fait preuve d'audace, d'autant plus que si ces prescriptions contraignantes ne concernent pour l'instant que les seuls chats, chiens et équidés, elles peuvent être facilement applicables aux autres espèces par voie de règlement. En 2008, le législateur suisse ${ }^{37}$ avait déjà retenu l'attention en obligeant les propriétaires à posséder plusieurs individus d'une même espèce ${ }^{38}$ et à assurer hebdomadairement à leur chien des contacts avec des humains et si possible d'autres chiens ${ }^{39}$.

En dernier lieu, l'analyse comparative intéresse les dispositions du chapitre $8^{40}$ qui fixent le niveau des sanctions encourues en cas d'atteinte au bien-être ou à la sécurité de l'animal. Tout acte ou omission plaçant un animal en situation de détresse est passible pour une première infraction d'une amende s'échelonnant de 250 à 250000 dollars. En cas de récidive les peines peuvent être doublées ou triplées et l'auteur de l'infraction encourt également une peine maximale d'emprisonnement de 18 mois. Ces dispositions de la Loi sur le bien-être et la sécurité de l'animal complètent le code criminel qui sanctionne les actes de cruauté envers les animaux par une peine d'emprisonnement maximale de 5 ans ou sur déclaration de culpabilité d'une amende maximale de dix mille dollars et/ou d'un emprisonnement maximal de 18 mois $^{41}$. Les peines encourues pour acte de cruauté varient fortement d'un pays à l'autre. Ainsi l'amende peut s'élever à 250 $000 €$ en Irlande, $100000 €$ en Espagne, $50000 €$ en Allemagne, $30000 €$ en France, 20 $500 €$ aux Pays-Bas, $7500 €$ en Autriche, 3000 Francs en Suisse, $2000 €$ en Belgique et au Luxembourg, 500€ en Bulgarie et $100000 €$ pesos en Colombie. Quant aux peines d'emprisonnement elles s'échelonnent de 5 ans en Irlande, 4 ans en Finlande, 3 ans en Suisse et en Allemagne, 2 ans en France et au Portugal, 6 mois en Belgique, Luxembourg

\footnotetext{
${ }^{37}$ Ordonnance du 23 avril 2008 sur la protection des animaux.

${ }^{38}$ Les espèces concernées sont notamment les yacks, chevreaux, lamas et alpagas, jeunes chevaux, lapereaux.

${ }^{39}$ Article 70 de l'ordonnance du 23 avril 2008 sur la protection des animaux.

${ }^{40}$ Articles 65 et suivants.

${ }^{41}$ L'article 445-1 du code criminel sanctionne les actes de cruauté visant à infliger une douleur, souffrance ou blessure sans nécessité à tout animal ou oiseau.
} 
et Colombie et 3 mois aux Pays-Bas. Le législateur québécois n'a donc pas suivi la ligne très dure du législateur irlandais en assortissant à un niveau élevé de sanction pécuniaire une peine privative de liberté de plusieurs années.

L'étude des nouvelles dispositions québécoises à l'aune d'autres législations nationales autorise à considérer que le Québec s'est extrait de la catégorie peu enviable des Etats les plus défavorables aux animaux. Pour autant, de nombreuses évolutions sont encore nécessaires pour consacrer une nouvelle catégorie juridique qui ne se confonde ni avec les choses ni avec les personnes physiques. A cette fin, deux axes qui émergent dans certains Etats peuvent être suivis : la reconnaissance d'une personnalité animale et la codification du régime juridique de l'animal. La reconnaissance d'une personnalité non humaine résulte notamment de décisions juridictionnelles telles que celles rendues par la Cour de Cassation argentine qui a attribué la qualité de sujet non-humain à une femelle orangoutan détenu en captivité au zoo de Buenos Aires ${ }^{42}$ et par la Haute Cour de l'État himalayen de l'Uttarakhand qui dans une décision du 4 juillet 2018 a déclaré que tous les individus composant le règne animal devaient être considérés comme des entités juridiques. Quant à l'élaboration d'un code animal contenant l'ensemble des dispositions applicables aux animaux notamment en matière de bien-être et de lutte contre la cruauté il est possible de s'inspirer du modèle belge. Le code wallon du bien-être animal, approuvé par le gouvernement et en attente d'un vote par les députés à l'automne 2018, comporte au sein de 109 articles des dispositions relatives à la détention des animaux, aux pratiques interdites et aux interventions autorisées sur les animaux, au commerce des animaux, au transport et à l'introduction d'animaux sur le territoire, à la mise à mort d'animaux, aux

${ }^{42}$ Suite à une action intentée sur la fondement de l'habeas corpus, la Cour de cassation a rendu le 21 décembre 2014 un arrêt dans lequel elle attribue la qualité de personne nonhumaine à laquelle on doit garantir au moins trois de ses droits basiques fondamentaux, la vie, la liberté et le droit de ne pas être maltraitée physiquement, ni psychologiquement. 
expériences sur animaux, aux infractions en matière de bien-être animal (contrôle, recherche, constatation, poursuite, répression, réparation). Le chemin est donc tracé, il ne reste plus qu'à le suivre. 